\title{
Validation d'une technique de dosage multiélémentaire des métaux et métalloïdes dans les ongles par ICP-MS. Valeurs usuelles chez 130 sujets volontaires
}

\section{ICP-MS metal and metalloid multielement analysis validation in fingernail: frequent values in 130 healthy volunteers}

\section{Jean-Pierre GOULLÉ(1)*, Lö̈c MAHIEU(1), Élodie SAUSSEREAU ${ }^{(1)}$, Daniel BOUIGE ${ }^{(2)}$, Sophie GROENWONT ${ }^{(1)}$, Christian LACROIX ${ }^{(1)}$}

(1) Laboratoire de Pharmacocinétique et de Toxicologie Cliniques, Groupe Hospitalier du Havre, BP 24, 76083 LE HAVRE CEDEX

(2) Laboratoire de Biochimie, Groupe Hospitalier du Havre, BP 24, 76083 LE HAVRE CEDEX

*Auteur à qui adresser la correspondance : Jean-Pierre GOULLÉ, Laboratoire de Pharmacocinétique et de

Toxicologie Cliniques, Groupe Hospitalier du Havre, BP 24, 76083 LE HAVRE CEDEX, France Tél : 0232733223 - Fax : 0232733238 - E-mail : jgoulle@ch-havre.fr

Ce travail a été présenté au $\mathrm{XV}^{\mathrm{eme}}$ Congrès annuel de la SFTA, Paris

(Reçu le 2 juillet 2007 ; accepté le 18 juillet 2007)

\section{RÉSUMÉ}

Nous décrivons une technique de dosage simultané de trente deux éléments minéraux dans les ongles, par plasma à couplage inductif relié à un détecteur de masse (ICP-MS). L'appareil utilisé est un spectromètre X7CCT Thermo Elemental, sans la cellule dynamique de réaction. Cent trente volontaires des deux sexes, indemnes de toute affection et sans traitement médical constituent les témoins. Des fragments d'ongles sont recueillis lors de la coupe de ceux-ci. Après décontamination de $20 \mathrm{mg}$ d'ongles, puis minéralisa-

\section{SUMMARY}

A thirty-two metal and metalloid quantification method for fingernail using inductively coupled plasma mass spectrometry (ICP-MS), is presented. The ICP-MS measurements were performed using a Thermo Elemental X7CCT series and Plasma Lab ${ }^{\circledast}$ software without the dynamic reaction cell. Fingernails from one hundred and thirty healthy volunteers, of both sexes, were collected. Twenty mg fingernails were acid mineralized after a decontamination procedure, and thirty-two metals and metalloids were simultaneously quan- 
tion acide, trente deux métaux et métallö̈des sont quantifiés simultanément par dilution de la solution acide obtenue. L'étalonnage est réalisé en milieu aqueux. Les dosages sont validés pour les trente deux éléments suivants : $\mathrm{Li}, \mathrm{Be}, \mathrm{B}, \mathrm{Al}$, V, Cr, Mn, Co, Ni, Cu, Zn, Ga, Ge, As, Se, Rb, Sr, Mo, Pd, Ag, $\mathrm{Cd}, \mathrm{Sn}, \mathrm{Sb}, \mathrm{Te}, \mathrm{Ba}, \mathrm{W}, \mathrm{Pt}, \mathrm{Hg}, \mathrm{Tl}, \mathrm{Pb}, \mathrm{Bi}, \mathrm{U}$. Les coefficients de régression sont supérieurs à 0,999 dans la gamme de linéarité considérée. Les limites de détection s'échelonnent de $0,04 \mathrm{pg} / \mathrm{mg}$ ou $\mathrm{ng} / \mathrm{g}(U)$ à $0,1 \mathrm{ng} / \mathrm{mg}$ ou $\mu \mathrm{g} / \mathrm{g}$ (B). Les résultats obtenus sont présentés et discutés à la lumière des concentrations publiées antérieurement. Les progrès récents de l'ICP-MS constituent un apport déterminant en biologie clinique et plus particulièrement dans le domaine de l'analyse toxicologique en raison de la puissance de l'outil. Son application à l'analyse multiélémentaire des métaux dans les ongles offre des perspectives complémentaires à l'analyse des ongles pour la recherche d'expositions au long cours ou d'intoxications chroniques. De plus, en raison de la structure particulière de l'ongle, ce phanère pourrait être proposé pour la surveillance professionnelle, environnementale ou domestique aux éléments métalliques, ainsi que pour la réalisation d'études épidémiologiques.

\section{MOTS-CLÉS}

ICP-MS, métaux, métalloüdes, ongles.

\section{Introduction}

Au cours des dernières années, l'analyse des xénobiotiques dans des matrices kératinisées comme les cheveux (1) ou les ongles (2) s'est développée en complément de l'analyse des milieux biologiques traditionnels que sont les urines ou le sang. Alors que le sang reflète la concentration en xénobiotiques à un instant précis et les urines sur une période tout au plus de quelques jours, les cheveux et les ongles sont le témoin de l'exposition aux substances étrangères durant plusieurs semaines ou mois. En ce qui concerne les cheveux, de nombreuses applications ont été proposées pour des xénobiotiques variés, médicaments ou drogues en toxicologie médico-légale (1) puis en toxicologie clinique (1). Le dosage des métaux et métalloïdes dans les cheveux s'est révélé relativement décevant en particulier dans le cadre de la surveillance d'expositions professionnelles (1), les applications essentielles étant limitées à quelques éléments :

- l'arsenic, à la recherche d'un arsénicisme hydrique lié à la contamination naturelle majeure des nappes phréatiques (3) ou d'un empoisonnement criminel (4) ;

- le mercure, pour apprécier l'exposition alimentaire au méthylmercure dans les régions du globe polluées par l'activité de l'homme, principalement l'extraction de l'or (5). Le mercure est le seul élément pour lequel une relation a pu être établie entre la concentration sangui- tified after acid dilution following water calibration. $\mathrm{Li}, \mathrm{Be}$, $B, \mathrm{Al}, \mathrm{V}, \mathrm{Cr}, \mathrm{Mn}, \mathrm{Co}, \mathrm{Ni}, \mathrm{Cu}, \mathrm{Zn}, \mathrm{Ga}, \mathrm{Ge}, \mathrm{As}, \mathrm{Se}, \mathrm{Rb}, \mathrm{Sr}, \mathrm{Mo}$, $\mathrm{Pd}, \mathrm{Ag}, \mathrm{Cd}, \mathrm{Sn}, \mathrm{Sb}, \mathrm{Te}, \mathrm{Ba}, \mathrm{W}, \mathrm{Pt}, \mathrm{Hg}, \mathrm{Tl}, \mathrm{Pb}, \mathrm{Bi}, \mathrm{U}$ fingernail determinations could be validated. Linearity was excellent and the correlation coefficients were above 0.999. Detection limits ranged from $0.04 \mathrm{pg} / \mathrm{mg}$ or $\mathrm{ng} / \mathrm{g}(U)$ to $0.1 \mathrm{ng} / \mathrm{mg}$ or $\mu g / g(B)$. Results are presented and compared to published multielement data. ICP-MS analysis has made significant advances in the field of clinical biology, particularly in toxicology. This is due to the use of extremely effective equipment that contributes to improved clinical, forensic, toxicological diagnosis which in turn permits greater control of industrial or environmental exposure, and for epidemiologic studies.

\section{KEY-WORDS}

ICP-MS, metals, metalloids, fingernails.

ne et la concentration capillaire suite à la consommation de méthylmercure (6). Le mercure se concentre dans les cheveux et sa teneur y est 250 fois plus élevée que dans le sang, ce facteur est reconnu et validé par l'OMS (7) ;

- le thallium, pour mettre en évidence un empoisonnement criminel (8) ;

- de manière plus ponctuelle, d'autres éléments pour confirmer une exposition dans des contextes variés.

L'intérêt du dosage des métaux et métalloïdes dans les cheveux, même s'il est limité, a malgré tout retrouvé un regain d'intérêt grâce à deux nouveaux apports : le dosage multiélémentaire simultané par spectrométrie d'émission à plasma induit relié à un détecteur de masse (ICP-MS) d'un grand nombre d'éléments (9) et le couplage de l'ICP-MS à la chromatographie liquide haute performance qui permet de réaliser la spéciation de certains éléments (10). Il nous a semblé intéressant d'appliquer ces nouvelles possibilités analytiques dans un premier temps au dosage multiélémentaire de métaux et métalloïdes dans les ongles dans la mesure où ceux-ci sont moins soumis à la contamination externe que les cheveux, en particulier dans le cadre d'expositions professionnelles.

L'ongle est constitué de couches de cellules kératinisées formées au niveau de la matrice, tissu épidermique hautement prolifératif (2). Comme le stratum corneum, la kératine cytoplasmique est partiellement cristalline 
et partiellement amorphe. L'ongle recouvre le lit unguéal qui est un tissu mou ; entre les deux, les cellules du lit unguéal migrent de manière distale dans l'ongle au fur et à mesure de leur naissance au niveau de la marge libre. La kératinisation de l'ongle dans la matrice s'effectue simultanément sur ses faces dorsale et ventrale, ultime étape de sa formation. Les fibres de kératine sont principalement orientées de manière perpendiculaire à l'axe de progression de l'ongle mais également dans le même sens que celui de la pousse assurant un lien très fort entre les couches. La structure finale est si dense qu'il n'y a pas d'exfoliation. La formation des ongles s'effectue dans deux directions, la longueur et l'épaisseur. La première, constituée par le développement de la matrice progresse à une vitesse linéaire moyenne voisine de $0,1 \mathrm{~mm}$ par jour pour les ongles de la main $(11,12)$ et de 0,03 à $0,04 \mathrm{~mm}$ par jour pour les ongles de pied (12). Cette vitesse diminue avec l'âge, le froid, la dénutrition et elle augmente chez les rongeurs d'ongles. Celle-ci est la même chez l'homme et chez la femme. La seconde direction permet de donner à l'ongle son épaisseur définitive grâce à la formation de couches ventrales au niveau du lit unguéal pendant la phase de croissance distale entre la lunule et la marge libre. La vitesse de pousse dans l'épaisseur est faible avec une valeur moyenne de $0,027 \mathrm{~mm} / \mathrm{mm}$ de longueur produite pendant la phase distale (13).

En ce qui concerne les xénobiotiques, ceux-ci sont incorporés dans les ongles par un double mécanisme :

- dépôt des substances véhiculées dans l'ongle qui se forme par le flux sanguin au niveau de la matrice ;

- incorporation jusqu'au début de la marge libre à partir de la lunule via le lit de l'ongle.

Compte tenu de la vitesse de pousse, la présence d'un xénobiotique lors de la coupe des ongles correspond à une exposition remontant à une période de 3 à 5 mois pour les ongles de la main et davantage pour les ongles des pieds.

\section{Matériel et méthodes}

Il s'agit d'une torche à plasma de type ThermoElectron $\mathrm{X}$ Series couplée à un spectromètre de masse, modèle X7/CCT (ThermoElectron, Courtaboeuf, France). L'appareil est équipé d'une torche en quartz de 1,5 mm, d'un nébuliseur concentrique en verre borosilicaté de $1 \mathrm{~mL}$ (type Meinhard de Marque Glass Expansion Référence ThermoElemental 1201318) avec un débit d'échantillon de $0,85 \mathrm{~mL} / \mathrm{min}$, d'une chambre simple de nébulisation à bille d'impact en quartz munie d'un refroidisseur à effet Peltier régulant sa température à $3^{\circ} \mathrm{C}$, et d'un passeur d'échantillons de type CETAC
ASX-510. L'ensemble des acquisitions est enregistré sur une station informatique dotée du logiciel d'analyse PlasmaLab version 2.0 sous Windows NT. Les paramètres instrumentaux sont les suivants : puissance de la torche $1200 \mathrm{~W}$; débits d'argon : plasmagène $15 \mathrm{~L} / \mathrm{min}$, nébuliseur $0,95 \mathrm{~L} / \mathrm{min}$, auxiliaire $0,66 \mathrm{~L} / \mathrm{min}$; interface : cônes échantillonneur et écorceur en nickel de diamètres respectifs de $1 \mathrm{~mm}$ et de $0,4 \mathrm{~mm}$; vide au niveau de l'interface : 1,9 mbar et du quadripôle : $1,6.10^{-7}$ mbar. Les réactifs, de qualité suprapur pour analyses de traces et les solutions étalon de métaux proviennent de chez Merck (Darmstadt, Allemagne) et CPI (Amsterdam, Hollande). Après décontamination par de l'acétone et de l'eau tiède, $20 \mathrm{mg}$ d'ongles sont minéralisés par $200 \mu \mathrm{L}$ d'acide nitrique suprapur pendant une heure à $70^{\circ} \mathrm{C}$ dans un tube bouché (soit $10 \mathrm{mg}$ pour $100 \mu \mathrm{L}$ ). Après refroidissement, à $100 \mu \mathrm{L}$ de la solution acide obtenue (il est donc possible de réduire la prise d'essai à $10 \mathrm{mg}$ ), on ajoute $100 \mu \mathrm{L}$ d'acide nitrique à $2 \%$ et $3800 \mu \mathrm{L}$ de diluant $(0,5 \%$ de butanol, $1 \%$ d'acide nitrique, In et Rh 1 ppb, 0,01\% triton). Trente deux éléments sont quantifiés simultanément dans les ongles : lithium, béryllium, bore, aluminium, vanadium, chrome, manganèse, cobalt, nickel, cuivre, zinc, gallium, germanium, arsenic, sélénium, rubidium, strontium, molybdène, palladium, argent, cadmium, étain, antimoine, tellure, baryum, tungstène, platine, mercure, thallium, plomb, bismuth, uranium. L'étalonnage est réalisé en milieu aqueux. Le protocole de validation est celui décrit dans un travail précédent (9). En l'absence de matériel certifié, la validité des résultats obtenus est contrôlée par la participation au programme de comparaison inter laboratoires organisé par l'institut national de santé publique du Québec (Sainte Foy, Canada), qui a comporté un exercice récent ponctuel de dosage dans une poudre d'ongles comprenant 23 éléments dont 14 ont été fortifiés.

\section{Résultats}

Les paramètres de la procédure de validation : élément ainsi que l'isotope quantifié, la linéarité, la limite de détection (LOD), la limite de quantification (LOQ), la répétabilité et la reproductibilité sont regroupés dans le tableau I. Pour les 32 éléments étudiés, le coefficient de corrélation reliant les concentrations et les signaux mesurés est supérieur à 0,999 . Les limites de détection obtenues pour les éléments les plus sensibles atteignent quelques dizaines de $\mathrm{fg} / \mathrm{mg}$ ou $\mathrm{pg} / \mathrm{g}$. Ainsi les limites de détection s'échelonnent de $0,04 \mathrm{pg} / \mathrm{mg}$ soit $0,04 \mathrm{ng} / \mathrm{g}$ pour l'uranium à $0,1 \mathrm{ng} / \mathrm{mg}$ soit $0,1 \mu \mathrm{g} / \mathrm{g}$ pour le bore. En ce qui concerne la répétabilité celle-ci est toujours inférieure à 3,9\% à l'exception du lithium 
Tableau I : Linéarité, limite de détection (LOD), limite de quantification (LOQ), répétabilité et reproductibilité.

\begin{tabular}{|c|c|c|c|c|c|}
\hline $\begin{array}{l}\text { Élément } \\
\text { isotope }\end{array}$ & $\begin{array}{c}\text { Linéarité } \\
\mathbf{r}\end{array}$ & $\begin{array}{l}\text { Limite de détection } \\
\text { en } \mathrm{ng} / \mathrm{mg}=\mu \mathrm{g} / \mathrm{g}\end{array}$ & $\begin{array}{l}\text { Limite de quantification } \\
\text { en } \mathrm{ng} / \mathrm{mg}=\mu \mathrm{g} / \mathrm{g}\end{array}$ & $\begin{array}{l}\text { Répétabilité } \\
\text { CV \% }\end{array}$ & $\begin{array}{l}\text { Reproductibilité } \\
\text { CV \% }\end{array}$ \\
\hline $7 \mathrm{Li}$ & 0,9999 & 0,002 & 0,007 & 6,5 & 6,1 \\
\hline 9Be & 0,9998 & 0,002 & 0,007 & 3,9 & 8,8 \\
\hline 11B & 0,9991 & 0,14 & 0,46 & 3,6 & 8,9 \\
\hline 27AI & 0,9993 & 0,02 & 0,08 & 2,3 & 7,7 \\
\hline $51 \mathrm{~V}$ & 0,9998 & 0,001 & 0,003 & 1,7 & 9,0 \\
\hline $53 \mathrm{Cr}$ & 0,9999 & 0,06 & 0,20 & 3,5 & 9,3 \\
\hline $55 \mathrm{Mn}$ & 0,9996 & 0,001 & 0,004 & 1,7 & 6,6 \\
\hline 59Co & 0,9998 & 0,0003 & 0,001 & 2,3 & 7,9 \\
\hline $60 \mathrm{Ni}$ & 0,9998 & 0,01 & 0,05 & 1,8 & 6,4 \\
\hline $65 \mathrm{Cu}$ & 0,9999 & 0,01 & 0,03 & 1,3 & 10,4 \\
\hline $66 \mathrm{Zn}$ & 0,9996 & 0,01 & 0,04 & 1,1 & 8,1 \\
\hline 69Ga & 0,9998 & 0,0003 & 0,0009 & 2,2 & 8,9 \\
\hline 74Ge & 0,9999 & 0,001 & 0,002 & 1,8 & 7,6 \\
\hline 75As & 0,9997 & 0,01 & 0,02 & 3,5 & 6,4 \\
\hline $82 \mathrm{Se}$ & 0,9997 & 0,02 & 0,06 & 2,6 & 7,8 \\
\hline 85Rb & 0,9995 & 0,0003 & 0,001 & 2,0 & 5,8 \\
\hline $88 \mathrm{Sr}$ & 0,9995 & 0,0002 & 0,0007 & 1,0 & 7,0 \\
\hline 98Mo & 0,9998 & 0,0004 & 0,001 & 3,9 & 8,2 \\
\hline 105Pd & 0,9995 & 0,001 & 0,003 & 2,9 & 22,3 \\
\hline 107Ag & 0,9998 & 0,0005 & 0,002 & 0,7 & 9,9 \\
\hline 111Cd & 0,9998 & 0,0003 & 0,0009 & 0,7 & 5,9 \\
\hline 118Sn & 0,9998 & 0,001 & 0,002 & 1,0 & 5,9 \\
\hline 121Sb & 0,9998 & 0,0003 & 0,001 & 1,0 & 5,2 \\
\hline 125Te & 0,9997 & 0,0006 & 0,002 & 6,7 & 6,1 \\
\hline 137Ba & 0,9998 & 0,001 & 0,003 & 0,8 & 5,5 \\
\hline $182 W$ & 0,9998 & 0,0002 & 0,001 & 2,1 & 7,2 \\
\hline 195Pt & 0,9999 & 0,0001 & 0,0002 & 1,5 & 6,2 \\
\hline $202 \mathrm{Hg}$ & 0,9986 & 0,004 & 0,013 & 0,4 & 9,5 \\
\hline 203TI & 0,9995 & 0,00005 & 0,0002 & 3,7 & 4,7 \\
\hline $208 \mathrm{~Pb}$ & 0,9997 & 0,0003 & 0,001 & 0,7 & 4,4 \\
\hline 209Bi & 0,9997 & 0,00008 & 0,003 & 1,4 & 5,3 \\
\hline $238 \mathrm{U}$ & 0,9998 & 0,00004 & 0,0002 & 2,0 & 7,2 \\
\hline
\end{tabular}

$(6,5 \%)$ et du tellure $(6,7 \%)$. La reproductibilité est toujours inférieure à $10 \%$ à l'exception du palladium $(22,3 \%)$. Les résultats obtenus lors de l'exercice ponctuel de contrôle de qualité externe canadien pour les 23 paramètres disponibles sont reportés dans le tableau II. Outre la concentration mesurée, le tableau indique, la concentration cible (médiane) calculée à partir des concentrations mesurées par les participants ainsi que le « $\mathrm{Z}$ score ». Celui-ci est établi après une troncature préalable à deux écarts-types. L'analyse statistique permet ensuite de calculer la médiane et l'écart type pour chaque élément. Les critères d'acceptabilité sont indiqués dans le tableau III. La qualité des résultats est appréciée par le calcul du Z-score qui s'effectue de la manière suivante :

$\mathrm{Z}=$ résultat rendu - concentration cible

écart attribué

Comme la concentration cible n'est pas connue, on utilise pour le calcul de Z-score, la concentration médiane calculée après troncature. Si la valeur absolue de $\mathrm{Z}$ est inférieure à 2 , les résultats sont satisfaisants ; entre 2 et 3 les résultats sont douteux; au-dessus de 3, ils ne 
Tableau II : Résultats obtenus lors de l'exercice ponctuel canadien sur de la poudre d'ongles (2006).

\begin{tabular}{|c|c|c|c|}
\hline Élément & $\begin{array}{l}\text { Concentration } \\
\text { Mesurée } \\
\text { (ng/mg) }\end{array}$ & $\begin{array}{l}\text { Concentration } \\
\text { cible } \\
\text { (ng/mg) }\end{array}$ & $Z$ score \\
\hline Ag* & 0,79 & 0,79 & 0,0 \\
\hline $\mathrm{Al}$ & 28,8 & 28,8 & 0,0 \\
\hline As* & 1,7 & 1,6 & 0,4 \\
\hline $\mathrm{Ba}$ & 1,7 & 1,9 & $-0,7$ \\
\hline $\mathrm{Be}^{*}$ & 0,59 & 0,53 & 0,7 \\
\hline $\mathrm{Cd}^{*}$ & 0,68 & 0,68 & 0,0 \\
\hline $\mathrm{Co}^{*}$ & 0,24 & 0,26 & $-0,2$ \\
\hline $\mathrm{Cr}$ & 1,3 & 1,7 & $-1,4$ \\
\hline $\mathrm{Cu}$ & 16,5 & 17,4 & $-0,8$ \\
\hline $\mathrm{Hg}^{*}$ & 7,6 & 8,7 & $-1,9$ \\
\hline Mn & 0,76 & 0,92 & $-1,1$ \\
\hline Mo* & 0,91 & 0,95 & $-0,2$ \\
\hline $\mathrm{Ni}$ & 2,8 & 2,5 & 1,0 \\
\hline $\mathbf{P b}$ & 6,2 & 7,3 & $-2,4$ \\
\hline $\mathbf{P t}^{*}$ & 0,43 & 0,43 & 0,0 \\
\hline $\mathrm{Sb}^{*}$ & 1,9 & 1,7 & 0,8 \\
\hline $\mathrm{Se}$ & 1,3 & 1,2 & 0,5 \\
\hline Sn* & 5,4 & 5,9 & $-1,3$ \\
\hline $\mathrm{Te}^{*}$ & 1,5 & 1,5 & $-0,2$ \\
\hline $\mathrm{Tl}^{*}$ & 0,35 & 0,36 & $-0,1$ \\
\hline $\mathrm{U}^{*}$ & 1,1 & 1,1 & 0,0 \\
\hline $\mathrm{V}^{*}$ & 0,70 & 0,76 & $-0,4$ \\
\hline $\mathrm{Zn}$ & 147 & 153 & $-0,5$ \\
\hline \multicolumn{4}{|c|}{ * Éléments fortifiés } \\
\hline
\end{tabular}

sont pas satisfaisants. Les concentrations usuelles sont établies chez 130 sujets des deux sexes. Pour chaque élément, la médiane et la dispersion des mesures du $5^{\mathrm{e}}$ au $95^{\mathrm{e}}$ percentile sont indiquées (Tableau IV).

A titre d'exemple, nous présentons les résultats obtenus lors du dosage du plomb dans les ongles chez des sujets d'une même famille à la suite d'une exposition domestique au plomb (Tableau V). Il s'agit de la découverte fortuite d'une plombémie élevée, non prescrite, chez une mère de 43 ans $(226 \mu \mathrm{g} / \mathrm{L}$ - normale $<63 \mu \mathrm{g} / \mathrm{L})$. Elle ne présente aucune symptomatologie clinique évocatrice d'une intoxication par ce métal. Le dosage du plomb dans les ongles est réalisé à distance de l'exposition, mais contemporain de celle-ci compte tenu de la vitesse de pousse. Il révèle une concentration de $33,8 \mu \mathrm{g} / \mathrm{g}$ (normale $<3,7 \mu \mathrm{g} / \mathrm{g}$ ). La plombémie mesurée 10 mois après l'exposition montre un retour lent à la normale $(82 \mu \mathrm{g} / \mathrm{L})$. L'analyse des ongles dix semaines plus tard le confirme $(5,1 \mu \mathrm{g} / \mathrm{g}$ et $4,5 \mu \mathrm{g} / \mathrm{g}$ respectivement dans les ongles de la main et du pied). Le père de 43 ans présente également une plombémie élevée $(191 \mu \mathrm{g} / \mathrm{L}$ - normale $<63 \mu \mathrm{g} / \mathrm{L})$ sans signe clinique. Le sujet se ronge les ongles, mais l'analyse des cheveux plusieurs mois après l'exposition révèle une
Tableau III : Critères d'acceptabilité pour le calcul du Z-score.

\begin{tabular}{|c|c|c|}
\hline $\begin{array}{c}\text { Concentration en } \\
\mathbf{n g} / \mathbf{m g} \text { ou } \boldsymbol{\mu g} \mathbf{g}\end{array}$ & $\begin{array}{c}\text { Erreur maximale } \\
\text { acceptée }\end{array}$ & Écart attribué \\
\hline$<0,5$ & $100 \%$ & $33,3 \%$ \\
\hline $0,5-2$ & $50 \%$ & $16,7 \%$ \\
\hline $2-5$ & $30 \%$ & $10 \%$ \\
\hline$>5$ & $20 \%$ & $6,7 \%$ \\
\hline
\end{tabular}

teneur en plomb augmentée $(45,1 \mu \mathrm{g} / \mathrm{g}$ - normale $<4,6$ $\mu \mathrm{g} / \mathrm{g})$. Les deux adolescents de 17 et 15 ans ont des concentrations sensiblement normales de plomb dans le sang et les ongles (Tableau V). En ce qui concerne les deux enfants les plus jeunes, âgés de 11 ans et 7 ans, qui n'étaient pas censés avoir été exposés, la découverte fortuite de concentrations très importantes dans les ongles (respectivement $89,1 \mu \mathrm{g} / \mathrm{g}$ et $185 \mu \mathrm{g} / \mathrm{g}$ - normale $<3,7 \mu \mathrm{g} / \mathrm{g}$ ) a conduit à la réalisation de dosages sanguins, certes tardifs par rapport à la période d'exposition, mais augmentés (respectivement $50 \mu \mathrm{g} / \mathrm{L}$ et $95 \mu \mathrm{g} / \mathrm{L}$ ). Ces résultats sont également en faveur d'une exposition au métal. L'enquête a révélé que le plomb provenait du décapage thermique de peinture sur des portes dans un habitat ancien, dans un espace pourtant ventilé. L'analyse d'écailles de la peinture qui contenait $26 \%$ de plomb a confirmé la nature domestique de cette exposition par inhalation.

\section{Discussion}

En ce qui concerne la validation du dosage des 32 éléments minéraux dans les ongles, les performances obtenues sont excellentes. La droite de la régression linéaire reliant les concentrations aux signaux mesurés est bonne puisque $\mathrm{R}^{2}$ est toujours au moins égal à 0,999 . Les limites de détection (LOD) sont particulièrement basses : de $40 \mathrm{fg} / \mathrm{mg}$ ou $40 \mathrm{pg} / \mathrm{g}$ soit 40 ppt pour l'uranium à 100 pg/mg ou ng/g pour le bore. Ces performances sont d'autant plus remarquables qu'elles correspondent à une prise d'essai de $10 \mathrm{mg}$ d'ongles. Pour le palladium, la médiocre reproductibilité obtenue (coefficient de variation à 22,3\%) a vraisemblablement pour origine l'emploi dans le même local d'un sel de palladium comme modificateur de matrice pour réaliser des dosages d'aluminium plasmatiques par spectrophotométrie d'absorption atomique électrothermique. Le dosage du palladium dans les ongles peut toutefois être utilisé dans un contexte d'exposition significative. En ce qui concerne le chrome, contrairement à d'autres matrices biologiques (sang total, plasma, urines) où le recours à une cellule de collision s'avère indispensable, en raison d'interférences spectrales, la validation de ce 
Tableau IV : Concentrations usuelles chez nos 130 sujets par ICP-MS comparées aux concentrations mesurées chez 96 sujets par ICP-MS avec secteur magnétique.

\begin{tabular}{|c|c|c|c|c|}
\hline & \multicolumn{2}{|c|}{ Nos résultats $-\mathbf{N}=\mathbf{1 3 0}$} & \multicolumn{2}{|c|}{ Rodushkin $-\mathrm{N}=96$ (14) } \\
\hline & $\begin{array}{l}\text { Médiane } \\
\mu \mathrm{g} / \mathrm{g}\end{array}$ & $\begin{array}{l}\text { Dispersion } \mu \mathrm{g} / \mathrm{g} \\
5^{\mathrm{e}}-95^{\mathrm{e}} \text { percentile }\end{array}$ & $\begin{array}{l}\text { Médiane } \\
\mu \mathrm{g} / \mathrm{g}\end{array}$ & $\begin{array}{l}\text { Dispersion }(\mu \mathrm{g} / \mathrm{g}) \\
5^{\mathrm{e}}-95^{\mathrm{e}} \text { percentile }\end{array}$ \\
\hline Lithium & 0,021 & $0,006-0,087$ & 0,053 & $0,13-0,255$ \\
\hline Béryllium & 0,004 & $0,001-0,011$ & 0,001 & $<0,001-0,007$ \\
\hline Bore & 0,43 & $0,07-3,14$ & 0,36 & $0,12-3,33$ \\
\hline Aluminium & 19,5 & $4,00-76,2$ & 32 & $12-137$ \\
\hline Vanadium & 0,051 & $0,027-0,114$ & 0,07 & $0,02-0,48$ \\
\hline Chrome & 0,38 & $0,03-1,89$ & 0,76 & $0,22-3,20$ \\
\hline Manganèse & 0,32 & $0,10-1,48$ & 0,65 & $0,19-3,30$ \\
\hline Cobalt & 0,020 & $0,009-0,069$ & 0,03 & $0,006-0,120$ \\
\hline Nickel & 0,94 & $0,22-8,34$ & 0,84 & $0,14-6,95$ \\
\hline Cuivre & 6,1 & $3,9-12,4$ & 7,6 & $4,2-17$ \\
\hline Zinc & 108 & $72-182$ & 116 & $80-191$ \\
\hline Gallium & 0,035 & $0,012-0,142$ & 0,009 & $0,003-0,053$ \\
\hline Germanium & 0,004 & $0,002-0,007$ & 0,005 & $<0,002-0,024$ \\
\hline Arsenic & 0,031 & $0,005-0,086$ & 0,22 & $0,07-1,1$ \\
\hline Sélénium & 0,62 & $0,44-0,91$ & 0,93 & $0,62-1,53$ \\
\hline Rubidium & 0,23 & $0,06-0,69$ & 0,20 & $0,04-1,30$ \\
\hline Strontium & 0,61 & $0,28-1,64$ & 0,39 & $0,17-1,39$ \\
\hline Molybdène & 0,012 & $0,005-0,034$ & 0,044 & $0,015-0,160$ \\
\hline Palladium & 0,015 & $0,006-0,048$ & 0,0006 & $<0,001-0,010$ \\
\hline Argent & 0,10 & $0,01-0,60$ & 0,11 & $0,02-1,76$ \\
\hline Cadmium & 0,028 & $0,009-0,196$ & 0,061 & $0,013-0,438$ \\
\hline Etain & 0,22 & $0,05-0,90$ & 0,48 & $0,11-2,56$ \\
\hline Antimoine & 0,039 & $0,012-0,196$ & 0,037 & $0,014-0,128$ \\
\hline Tellure & 0,0003 & $0,0003-0,0009$ & 0,0004 & $<0,0001-0,068$ \\
\hline Baryum & 0,66 & $0,21-3,07$ & 0,89 & $0,28-3,99$ \\
\hline Tungstène & 0,003 & $0,001-0,027$ & 0,015 & $0,003-0,053$ \\
\hline Platine & 0,0001 & $0,00005-0,0013$ & 0,0001 & $<0,0001-0,0011$ \\
\hline Mercure & 0,29 & $0,06-0,83$ & 0,10 & $0,03-0,31$ \\
\hline Thallium & 0,0004 & $0,0002-0,0012$ & 0,0012 & $0,0003-0,058$ \\
\hline Plomb & 0,52 & $0,10-3,71$ & 1,06 & $0,27-4,75$ \\
\hline Bismuth & 0,011 & $0,001-0,26$ & 0,021 & $0,004-0,54$ \\
\hline Uranium & 0,003 & $0,001-0,01$ & 0,008 & $0,002-0,05$ \\
\hline
\end{tabular}

paramètre dans les ongles n'a posé aucun problème, comme cela avait été le cas pour les cheveux avec de bonnes performances lors de l'exercice ponctuel interlaboratoires organisé par l'institut national de santé publique du Québec. Les résultats obtenus à l'occasion de ce contrôle ponctuel sont très bons avec un Z-score très légèrement supérieur à 2 pour le plomb $(2,4)$. Les concentrations usuelles mesurées chez nos 130 sujets appellent un certain nombre de commentaires.
L'analyse statistique des concentrations révèle une distribution soit de type gaussien soit de type log-gaussien avec pour la plupart des éléments quelques valeurs bien au-delà du $95^{\circ}$ percentile. Pour établir des concentrations normales pour chaque élément, on utilise communément la moyenne arithmétique et l'écart type. Cependant la présence de quelques valeurs supérieures au $95^{\mathrm{e}}$ percentile pèse parfois de manière significative sur cette moyenne, c'est la raison pour laquelle nous 
Tableau V : Exemple d'exposition familiale domestique au plomb.

\begin{tabular}{|c|c|c|c|c|}
\hline Sexe Age & Date & $\begin{array}{c}\text { Ongles } \\
\mathrm{N}<3,7 \mu \mathrm{gg} / \mathrm{g}\end{array}$ & $\begin{array}{c}\text { Sang total } \\
\mathrm{N} \text { adulte }<63 \mu \mathrm{g} / \mathrm{L}\end{array}$ & Signes cliniques \\
\hline \multirow{4}{*}{ F 43 ans } & 12.04 .06 & & 226 & \multirow{4}{*}{ Aucun } \\
\hline & 01.02 .07 & 33,8 & & \\
\hline & 01.03 .07 & & 82 & \\
\hline & 12.05 .07 & $\begin{array}{c}5,1 \\
\text { (pieds 4,5) }\end{array}$ & & \\
\hline \multirow[b]{2}{*}{ M 41 ans } & 15.05 .06 & & 191 & \multirow[b]{2}{*}{ Aucun } \\
\hline & 12.04 .07 & $\begin{array}{c}\text { Cheveux } \\
45,1 \mu \mathrm{g} / \mathrm{g} \\
\mathrm{N}<4,6 \mu \mathrm{g} / \mathrm{g}\end{array}$ & & \\
\hline \multirow{2}{*}{ F 17 ans } & 01.02 .07 & 4,3 & & \multirow{2}{*}{ Aucun } \\
\hline & 01.03 .07 & & 33 & \\
\hline \multirow[b]{2}{*}{ M 15 ans } & 01.03 .07 & & 39 & \multirow[b]{2}{*}{ Aucun } \\
\hline & 02.05 .07 & $\begin{array}{c}8,2 \\
\text { (pieds } 6,4 \text { ) }\end{array}$ & & \\
\hline \multirow[b]{3}{*}{ M 11 ans } & 01.02 .07 & 89,1 & & \multirow[b]{3}{*}{ Aucun } \\
\hline & 02.03 .07 & & 50 & \\
\hline & 13.05 .07 & $\begin{array}{c}19,1 \\
\text { (pieds } 19,2 \text { ) }\end{array}$ & & \\
\hline \multirow{4}{*}{ M 7 ans } & 01.02 .07 & 185 & & \multirow{4}{*}{ Aucun } \\
\hline & 01.03 .07 & & 95 & \\
\hline & 11.04 .07 & & 81 & \\
\hline & 02.05 .07 & $\begin{array}{c}20,4 \\
\text { (pieds } 46,8 \text { ) }\end{array}$ & & \\
\hline
\end{tabular}

utilisons plutôt la médiane et les percentiles, indicateurs beaucoup plus robustes aux valeurs extrêmes. La comparaison des résultats avec ceux publiés récemment par Rodushkin (14) par ICP-MS sur un appareil à secteur magnétique chez 96 sujets montre que nous obtenons des concentrations voisines pour la plupart des éléments. Pour douze d'entre eux les concentrations mesurées sont inférieures à celles rapportées par Rodushkin (Li, V, Cr, Mn, As, Mo, Cd, Sn, W, Tl, Bi, $\mathrm{U})$; pour dix sept elles sont relativement comparables (Be, B, Al, Co, Ni, Cu, Zn, Ge, Se, Rb, Sr, Ag, Sb, Te, $\mathrm{Ba}, \mathrm{Pt}, \mathrm{Pb})$; enfin pour trois éléments elles sont sensiblement plus élevées $(\mathrm{Ga}, \mathrm{Pd}, \mathrm{Hg})$. En ce qui concerne le gallium, une interférence avec le baryum est probable, alors que les concentrations plus élevées de palladium sont vraisemblablement en rapport avec l'emploi du palladium comme modificateur de matrice lors du dosage d'aluminium par spectrométrie d'absorption atomique électrothermique dans le même local. Quant au mercure la différence peut s'expliquer par une consommation plus importante de poisson dans la population examinée par rapport à celle étudiée par Rodushkin. Avec une concentration médiane de $0,29 \mu \mathrm{g} / \mathrm{g}$ de mercure dans les ongles, nos résultats sont très voisins de ceux publiés par Morton (15) et Samanta
(16) qui ont obtenu respectivement $0,24 \mu \mathrm{g} / \mathrm{g}$ et $0,39 \mu \mathrm{g} / \mathrm{g}$. A l'inverse la concentration médiane que nous obtenons pour l'arsenic $(0,031 \mu \mathrm{g} / \mathrm{g})$ est nettement plus faible que celle mesurée par Rodushkin $(0,223 \mu \mathrm{g} / \mathrm{g})$. Ceci est peut être dû à une faible exposition à de l'arsenic minéral, probablement d'origine alimentaire, car notre médiane est comparable à celle obtenue par Beane-Freeman chez 329 sujets $(0,040$ $\mu \mathrm{g} / \mathrm{g})(17)$.

La rétention de certains éléments comme l'arsenic dans des matrices kératinisées (cheveux, ongles) est connue de longue date. Cependant, l'analyse des ongles présente un certain nombre d'avantages par rapport aux cheveux. Le risque de contamination externe est plus faible et la vitesse de pousse plus régulière. Par ailleurs la quantité de mélanine contenue dans les cheveux influe de manière sensible sur l'incorporation des xénobiotiques. Enfin, l'analyse des cheveux peut être faussée par divers traitements cosmétiques tels la coloration, la décoloration et les permanentes voire les shampoings susceptibles d'éliminer une partie des xénobiotiques fixés. La contamination externe environnementale des cheveux a été clairement montrée par Anwar (18) chez des habitants d'une zone urbaine pour le plomb et le cadmium. En effet, ces sujets présentaient 
des concentrations plus élevées pour ces éléments dans les cheveux que les sujets d'une zone non urbaine alors que les concentrations dans les ongles des pieds étaient identiques. Pour mettre en évidence une exposition professionnelle, l'analyse des ongles de pied doit être privilégiée. Ainsi dans le cas du plomb, Barbosa (19) rappelle que les ongles de pied sont moins sensibles à la contamination que ceux de la main. Le dosage des métaux et métalloïdes dans les ongles par ICP-MS a été réalisé dans divers contextes. Ils concernent des examens effectués dans le cadre d'intoxications ou d'expositions à certains éléments :

- l'arsenic en médecine légale dans le cadre d'intoxications criminelles $(4,20)$;

- le thallium dans un cas d'homicide (21) et dans deux observations d'intoxication volontaire avec des concentrations respectives dans les ongles de $2,5 \mu \mathrm{g} / \mathrm{g}$ et de $1,7 \mu \mathrm{g} / \mathrm{g}(8)$;

- le plomb dans un cas d'intoxication mortelle qui a révélé après exhumation une concentration de $13,6 \mu \mathrm{g} / \mathrm{g}$ dans les ongles (22) ;

- le tungstène dans un cas d'intoxication par cet élément (23) ;

- les ongles de la main ont été proposés comme marqueurs biologiques d'exposition aux métaux dans différents contextes médicaux (24-26) ;

- ils ont été parfois étudiés dans le cas d'expositions environnementales (27-29) ;

- le dosage de métaux dans les ongles a également été suggéré comme marqueur d'exposition qualitative à certains métaux : cobalt, tungstène et tantale par exemple (30) ;

- l'exposition au mercure liée à l'emploi d'amalgames dentaires chez les dentistes a été étudiée. Joshi (31) montre que la concentration moyenne dans les ongles de pied est de $0,94 \mu \mathrm{g} / \mathrm{g}$ contre $0,54 \mu \mathrm{g} / \mathrm{g}$ dans la population générale. Morton (15) met en évidence des concentrations moyennes de mercure plus élevées dans les ongles de la mains des dentistes $(1,42 \mu \mathrm{g} / \mathrm{g})$ comparées aux témoins $(0,24 \mu \mathrm{g} / \mathrm{g})$ et aux ongles du pied de ces dentistes $(0,43 \mu \mathrm{g} / \mathrm{g})$ comparés aux témoins $(0,18 \mu \mathrm{g} / \mathrm{g})$.

La contamination de certaines régions du globe a également trouvé des applications au dosage de certains éléments dans les ongles. Ainsi, l'arsenicisme chronique sévit dans l'ouest du Bengale où plus de $60 \%$ de la population vit dans des zones dont l'eau des puits est polluée. Cette pollution affecte la santé de plusieurs millions d'habitants, elle est responsable de centaines de milliers de décès dus à des cancers de la peau, de l'estomac, du poumon, du foie et du rein. Ce que l'on s'accorde à considérer comme la plus grande catas- trophe écologique de tous les temps a fait l'objet de plusieurs publications récentes portant sur l'analyse dans les phanères dont les ongles :

- Anawar (32) dans une étude portant sur 10 districts du Bangladesh rappelle que $66 \%$ des échantillons d'eau analysés contiennent plus de $10 \mu \mathrm{g} / \mathrm{L}$ d'arsenic. Il cite les résultats d'analyses (33) réalisées quelques années plus tôt où les habitants de ces districts présentaient des concentrations augmentées d'arsenic dans les cheveux, dans les ongles et dans les urines (respectivement 96 $\%, 95 \%$ et $94 \%$ d'entre eux). Les concentrations mesurées dans les ongles sont très élevées (de $1,3 \mu \mathrm{g} / \mathrm{g}$ à $33,98 \mu \mathrm{g} / \mathrm{g}$ ) alors que les concentrations normales retenues par les auteurs sont comprises entre 0,43 et $1,08 \mu \mathrm{g} / \mathrm{g}$ (34), concentrations qui sont très supérieures à celles que nous avons établies dans ce travail $(<0,1$ $\mu \mathrm{g} / \mathrm{g})$.

- Samanta (16) dans un travail portant sur 10 éléments chez 33 sujets montre, outre des augmentations très importantes d'arsenic dans les ongles de la main (concentration médiane de $4,73 \mu \mathrm{g} / \mathrm{g}$ ), mais également d'autres éléments : plomb, nickel et manganèse avec des concentrations médianes respectives de $7,47 \mu \mathrm{g} / \mathrm{g}$ $2,98 \mu \mathrm{g} / \mathrm{g}$ et $24,51 \mu \mathrm{g} / \mathrm{g}$.

- La validité du dosage de l'arsenic et du sélénium dans les ongles comme biomarqueur d'exposition a également fait l'objet d'une récente revue (35).

- La spéciation de certains éléments a également été étudiée. Elle fait appel à des couplages HPLC-ICP-MS ou GC-ICP-MS. Ainsi dans une zone contaminée par l'arsenic, Mandal (10) a montré à l'aide d'un couplage HPLC-ICP-MS que les ongles des mains contenaient en moyenne 58,6\% d'arsenic inorganique (III), 21,5\% d'arsenic inorganique (IV), 7,7 de MMA (V), 9,2 \% de DMA (III) et 3,0 \% de DMA (IV). Les formes non toxiques d'arsenic, contenues parfois en grande quantité dans les produits de la mer, arsénobétaïne et arsenocholine en particulier sont rapidement éliminées en l'état dans les urines et ne sont pas retrouvées dans les phanères (36).

La mesure des isotopes de certains éléments peut s'avérer intéressante :

- pour l'uranium, le rapport isotopique ${ }^{234} \mathrm{U} / 238 \mathrm{U}$ est un bon indicateur de l'exposition à cet élément (37) ;

- pour le plomb, les rapports isotopiques ${ }^{206} \mathrm{~Pb} /{ }^{207} \mathrm{~Pb}$ comparés aux rapports ${ }^{208} \mathrm{~Pb} /{ }^{207} \mathrm{~Pb}$ dans les ongles, les cheveux et une peinture utilisée permettent dans un cas d'exposition au plomb d'attribuer à la peinture utilisée l'élévation du plomb dans ces deux matrices biologiques (14). 


\section{Conclusion}

Cette nouvelle application de l'ICP-MS consacrée au dosage des métaux confirme s'il en était besoin qu'il s'agit d'une technique spécifique et d'une grande sensibilité. Elle permet avec un échantillon réduit d'ongles, de quantifier une trentaine de métaux et métalloïdes simultanément et dans un délai relativement court. La méthode décrite est parfaitement adaptée aux dosages en série. A partir d'un prélèvement non invasif, il est possible d'explorer un grand nombre d'éléments. Elle offre des perspectives nouvelles d'exploration dans des domaines variés de la toxicologie : clinique, judiciaire, professionnelle, environnementale ; à la recherche d'intoxications ou d'expositions au long cours. Toutes les caractéristiques de cette nouvelle application en font également un outil idéal pour la réalisation d'études épidémiologiques.

Cet équipement a été acquis grâce à un financement de l'association pour la Fondation Charles Nicolle - Rouen - France

\section{Références}

1. Kintz P. Analytical and practical aspects of drug testing in hair. ed. Taylor \& Francis : CRC Press , 2006, 382 p.

2. Palmieri A., Pichini S, Pacifici R, Zuccaro P, Lopez A. Drugs in nails: physiology, pharmacokinetics and forensic toxicology. Clin. Pharmacokinet. 2000 ; 38 : 95-110.

3. Goullé J.P. Metals. In Kintz P., ed. Taylor \& Francis. Analytical and practical aspects of drug testing in hair. CRC Press, 2006 ; 343-70.

4. Goullé J.P., Mahieu L., Kintz P. The murder weapon was found in the hair. Ann. Toxicol. Anal. 2005 ; 4 : 243-6.

5. Horvat M., Nolde N., Fajon V., Jereb V., Logar M., Logen S., Jacimovic R., Falnoga I., Liya Q., Faganeli J., Drobne D. Total mercury, methylmercury and selenium in mercury polluted areas in the province Guizhou, China. Sci. Total Environ. 2003 ; 304 : 231-56.

6. WHO (World Health Organisation) : Methylmercury. Environmental Health Criteria 101. Geneva. 1990.

7. Johnsson C., Schütz A., Sällsten G. Impact of consumption of freshwater fish on mercury levels in hair, blood, urine, and alveolar air. J. Toxicol. Environ. Health A. $2005 ; 68: 129-40$.

8. Mc Cormack J., Mc Kinney W. Thallium poisoning in group assassination attempt. Postgraduate Medecine. $1983 ; 74: 239-42$.

9. Goullé J.P., Mahieu L., Bonneau L., Lainé G., Bouige D., Lacroix C. Validation d'une technique de dosage multiélémentaire des métaux et métalloïdes dans les cheveux par ICP-MS. Valeurs de référence chez 45 témoins. Ann. Toxicol. Anal. $2005 ; 17: 97-103$.
10. Mandal B.K., Ogra Y., Suzuki K.T. Speciation of arsenic in human nail and hair from arsenic-affected area by HPLC-inductively coupled argon plasma mass spectrometry. Toxicol. Appl. Pharmacol. 2003 ; 189 : 73-83.

11. Le Gros C. WE, Dudley B. LH. Studies in nail growth. Br. J. Dematol. $1938 ; 50: 221-35$.

12. Bean W. B. A note on fingernail growth. J. Invest Dermatol. 1953 ; 20 : 27-31.

13. Johnson M. Shuster S. Continuous formation of nail along the bed. Br. J. Dermatol. $1993 ; 128$ : 277-80.

14. Rodushkin I., Axelsson M.D. Application of double focusing sector field ICP-MS for multielemental characterization of human hair and nails. Part II. A stydy of the inhabitants of northern Sweden. Sci. Total Environ. 2000 ; $262: 21-36$.

15. Morton J., Mason H.J, Ritchie K. A. White M. Comparison of hair, nails and urine for biological monitoring of low level inorganic mercury exposure in dental workers. Biomarkers. 2004 ; 9 : 47-55.

16. Samanta G., Sharma R., Roychowdhury T., Chakraborti D. Arsenic and other element in hair, nails ans skin-scales of arsenic victims in West Bengal, India. Sci Total Environ. 2004 ; 326 : 33-47.

17. Beane-Freeman L. E., Denis L. K., Lynch C. F., Thorne P. S., Just C. L. Total arsenic content and cutaneous melanoma in Iowa. Am. J. Epidemiol. 2004 ; 160 : 679-87.

18. Anwar M. Arsenic, cadmium and lead levels in hair and toenail samples in pakistan. Environ. Sci. $2005 ; 12: 71-86$.

19. Barbosa F. Jr., Tanus-Santos J. E., Gerlach R. F., Parsons P. J. A critical review of biomarkers used for monitoring human exposure to lead : advantages, limitations, and future needs. Environ. Health Perspect. 2005 ; 12 : 1669-74.

20. Daniel C.R. 3rd, Piraccini B. M., Tosti A. The nail and hair in forensic science. J. Am. Acad. Dermatol. 2004 ; 50 : 258-61.

21. Hann S., Latkoczy C., Bereuter T.L. Prohaska T., Stingeder G., Reiter C. Reconstruction of a case of thallium poisoning using LA-ICP-SFMS. Int. J. Legal Med. $2005 ; 119: 35-9$.

22. Lech T. Exhumation examination to confirm suspicion of fatal lead poisoning. Forensic Sci. Int. 2006 ; 158 : 219-23.

23. Marquet P., François B., Lotfi H., Turcant A., Debord J., Nedelec G., Lachatre G. Tungsten determination in biological fluids, hair and nails by plasma emission spectrometry in a case of severe acute intoxication in man. J. Forensic Sci. 1997 ; 42 : 527-30.

24. Mehra R., Juneja M. Fingernails as biological indices of metal exposure. J. Biosci. $2005 ; 30: 253-7$.

25. Rajpathak S., Rimm E. B., Li T., Morris J. S., Stampfer M. J., Willett W. C., Hu F. B. Lower toenail chromium in men with diatetes and cardiovascular disease compared with healthy men. Diabetes Care. 2004 ; 27 : 2211-6.

26. Tang Y. R., Zhang S. Q., Xiong Y., Zhao Y., Fu H., Zhang H. P., Xiong K. M. Studies of five microelement contents in human serum, hair, and fingernails correlated with aged hypertension and coronary heart disease. Biol. Trace Elem. Res. 2003 ; 92 : 97-104. 
27. Nowak B., Chmielnicka J. Relationship of lead and cadmium to essential elements in hair, teeth, and nails of environmentally exposed people. Ecotoxicol. Environ. Saf. $2000 ; 46: 265-74$.

28. Slotnick M.J., Meliker J. R., AvRuskin G.A., Ghosh D., Nriagu J. O. Toenails as a biomarker of inorganic arsenic intake from drinking water and foods. J. Toxicol. Environ. Health A. $2007 ; 70$ : 148-58.

29. Wickre J. B., Folt C. L., Sturup S., Karagas M. R. Environmental exposure and fingernail analysis of arsenic and mercury in children and adults in a Nicaraguan gold mining community. Arch. Environ. Health. 2004 ; 59 : 400-9.

30. Sabbioni E. Minoia C., Pietra R., Mosconi G., Forni A., Scansetti G. Metal determinations in biological specimens of diseased and non-diseased hard metal workers. Sci. Total Environ. 1994 ; 150 : 41-54.

31. Joshi A, Douglass C. W., Kim H. D., Joshipura K. J., Park M. C., Rimm E.B., Carino M. J. Garcia R. I., Morris J. S., Willett W. C. The relationship between amalgam restorations and mercury levels in male dentists and nondental health professionals. J. Public Health Dent. 2003 ; 63 : 5260 .

32. Anawar H. M., Akai J., Mostofa K.M.G, Safiullah S., Tareq S.M. Arsenic poisoning in groundwater health risk and geochemical sources in Bangladesh. Environment International. $2002 ; 27$ : 597-604.

33. DHC report. The arsenic disater and Dhaka Community Hospital, at the seminar on arsenic disaster in Bangladesh environment, Dhaka, Bangladesh. 1997 ; January 6.

34. Dhar R. K., Biswas B. K., Samanta G., Mandal B. K., Chakraborty D., Roy S., Jafar A., Islam A., Ara G., Kabir S., Khan A.W. Ahmed S. A., Hadi S. A. Groundwater arsenic calamity in Bangladesh. Curr. Sci . 1997 ; 73 : 4859.

35. J. Slotnick M., O. Nriagu J. Validity of human nails as a biomarker of arsenic and selenium exposure : A review. Environ. Research. 2006 ; 102 : 125-35.

36. National Research council's Subcommitte on Arsenic in Drinking Water, Arsenic In Drinking Water. National Academy Press. Washington. DC 1999; 177-92.

37. Karpas Z., Lorber A., Sela H., Paz-Tal O., Hagag Y. Kurttio P., Salonen L. Measurement of the 234U/238U ratio by MC-ICPMS in drinking water, hair, nails, and urine as an indicator of uranium exposure source. Health Phys. 200589 : 315-21. 